\title{
Shock-Wave Synthesis of Nanoparticles during Ion Sputtering
}

\author{
L. E. Rehn, R. C. Birtcher, S. E. Donnelly ${ }^{1}$, P. M. Baldo and L. Funk, \\ Materials Science Division, Argonne National Laboratory \\ Argonne, IL 60439, U.S.A. \\ ${ }^{1}$ Joule Physics Laboratory \\ University of Salford, \\ Salford M5 4WT, U.K.
}

\begin{abstract}
We report electron microscopy studies of nanoparticles ( $500 \leq \mathrm{n} \leq 10^{4}$, where $\mathrm{n}$ is the number of atoms in a given cluster) that are sputtered from the surface by high-energy ion impacts. Measurements of the sizes of these clusters yielded an inverse power-law distribution with an exponent of -2 that is independent of irradiating ion species and total sputtering yield. This inverse-square dependence indicates that these nanoclusters are produced when shock waves, generated by sub-surface displacement cascades, impact and ablate the surface. Such nanoparticles consist of simple fragments of the original surface, i.e., ones that have not undergone any large thermal excursion. As discussed below, this "ion ablation" technique should therefore be useful for synthesizing nanoparticles of a wide variety of alloy compositions and phases.
\end{abstract}

\section{INTRODUCTION}

The emission of intact clusters of atoms during ion sputtering was first reported more than 40 years ago [1]. This observation elicited considerable surprise [2 - 5] because the energies involved in the ion-target collisions that generate sputtered atoms are typically much larger, ranging up to many $\mathrm{keV}$, than the $1-2 \mathrm{eV}$ typical binding energies holding the clusters together. We recently reported [6] transmission electron microscopy (TEM) studies of the sizedistributions of the larger clusters $(>500)$ that are sputtered from the surface by high-energy ion impacts. These new measurements yielded a power-law size distribution with an exponent of -2 , independent of ion species and total sputtering yield. Such an inverse-square dependence revealed that the clusters are produced when shock waves, generated by sub-surface displacement cascades, impact and ablate the surface, as originally proposed in the model of Bitensky and Parilis [7]. Such nanoparticles consist of simple fragments of the original surface, i.e., ones that have not undergone any significant thermal excursion. As discussed in greater detail below, this ion-ablation technique should therefore be useful for synthesizing nanoparticles of a wide variety of alloy compositions and phases.

\section{EXPERIMENTAL DETAILS}

The experiments were performed as follows. Au films, $50 \pm 5 \mathrm{~nm}$ thick with a $<110>$ surface normal, were deposited by e-beam evaporation of 99.999 at.\% $\mathrm{Au}$ onto $\mathrm{NaCl}$ substrates held at a temperature of $350{ }^{\circ} \mathrm{C}$. Pieces of the evaporated Au films were floated off the $\mathrm{NaCl}$ in a 
water/alcohol mixture and sifted onto $\mathrm{Cu}$ TEM grids. The ion sputtering experiments were performed in the Hitachi H-9000 electron microscope operating at $300 \mathrm{kV}$, located in the IVEMTandem facility at Argonne National Laboratory [8]. The ion beam was oriented $30^{\circ}$ from the microscope axis, and the specimen was tilted $15^{\circ}$ toward the ion beam so that both ions and electrons were incident on the specimens at $15^{\circ}$ to the foil normal. The ions passed first through the 50-nm thick gold foil target pieces, then through a TEM transparent carbon-coated collector grid that was positioned approximately $30 \mu \mathrm{m}$ below the target foil. This transmission-sputtering geometry was chosen because it provided superior in-situ images of the collected particles. Although the size-distributions were not analyzed in detail, we did observe similar sized particles in a reflection geometry.

Nanoparticle synthesis was performed with a series of ions ( $\mathrm{Ne}, \mathrm{Ar}, \mathrm{Kr}, \mathrm{Xe}$ and $\mathrm{Au}$ ) of increasing atomic mass at energies between 400 and $500 \mathrm{keV}$. The target and collector foils were at room temperature and the ion flux was maintained between $(1-2) \times 10^{15}$ ions $/ \mathrm{m}^{2} / \mathrm{s}$. The beam current was monitored continuously during the sputtering experiments using an annular Faraday cage that was calibrated via a removable Faraday cup having an absolute accuracy estimated to be $\pm 10 \%$. The total ion fluence was kept low, less than $1 \mathrm{ion} / \mathrm{nm}^{2}$, in order to minimize any potential complications from either surface roughening, target thinning, or resputtering of collected particles.

\section{RESULTS AND DISCUSSION}

Bright-field TEM micrographs of nanoparticles collected on the carbon-coated grids following separate 400-keV Ne, $\mathrm{Kr}$ and $\mathrm{Xe}$ irradiations to fluences of approximately $10^{18}$ ions $/ \mathrm{m}^{2}$ are shown in Fig. 1. As can be readily seen in the micrographs, the sizes of the collected particles are broadly distributed, from just barely discernible images somewhat less than a nm in lateral dimension, up to particles as large as approximately $8 \mathrm{~nm}$ in extent. The TEM imaging conditions were adjusted so that the contrast in Fig. 1 arises primarily from differences in total thickness. Hence the fact that the larger particles are noticeably darker in the micrograph demonstrates that the particles are three-dimensional entities. The three-dimensional nature of the collected particles was subsequently confirmed from high-resolution TEM (HRTEM) images taken in a JEOL4000 microscope.
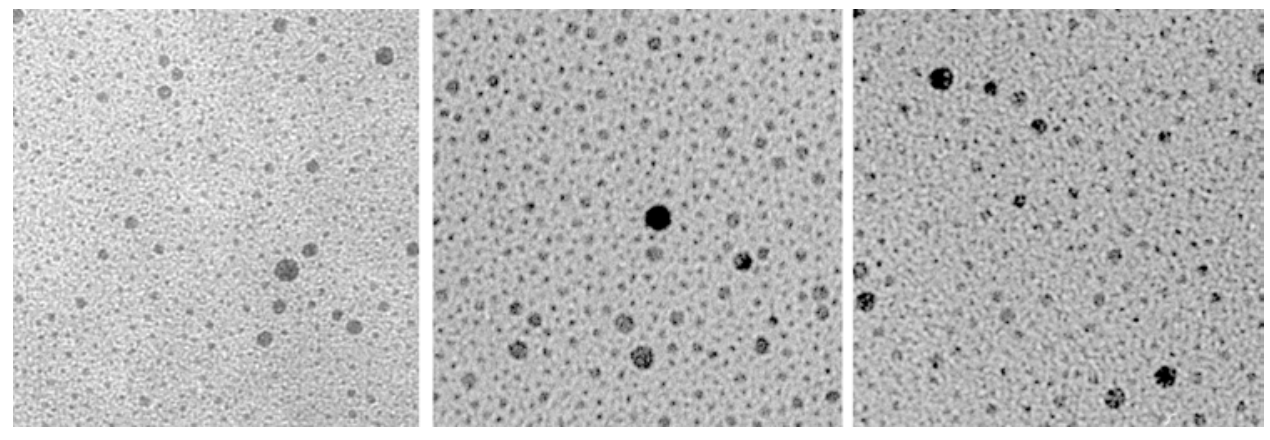

Fig. 1: Bright field TEM micrograph of Au nanoparticles collected on carbon-coated grids following 400-keV Ne, $\mathrm{Kr}$ and Xe irradiations to fluences of $1 \times 10^{18} \mathrm{ions} / \mathrm{m}^{2}$, respectively. Note 
that the larger particles are noticeably darker, demonstrating that the particles are threedimensional entities.

The particle size distributions, Y(n), i.e., the number of clusters containing $n$ atoms, were determined after removing the Au target foil as follows. First, an image similar to the ones shown in Fig. 1 was digitized using a Leafscan 45 at a final image resolution of 10 pixels $/ \mathrm{nm}$. Sizes of individual nanoparticles were obtained from the scanned images using the computer program Photoshop. The measured lateral dimensions were converted into the number of atoms for a given cluster by assuming that the particles were hemispheres with the density of bulk Au. All visible particles in an area of $\sim 10^{5} \mathrm{~nm}^{2}$ were sized, i.e., approximately 650 and 200 particles, respectively, for the $\mathrm{Au}$ and the $\mathrm{Ne}$ irradiations. The results were grouped into bins having 0.5 $\mathrm{nm}$ steps; the procedure was repeated following each irradiation. As reported previously [9], insitu video recordings showed that the sputtered clusters arrived at the collector foil wellseparated, both temporally and spatially. The total nanoparticle density determined by counting individual nanoparticles in the scanned images had a one-to-one correlation with the integrated arrival rates determined from the in-situ video recordings. Subsequent irradiation of the particles without a target foil did not produce particle growth. Taken together these findings demonstrate that size changes following deposition were insignificant.

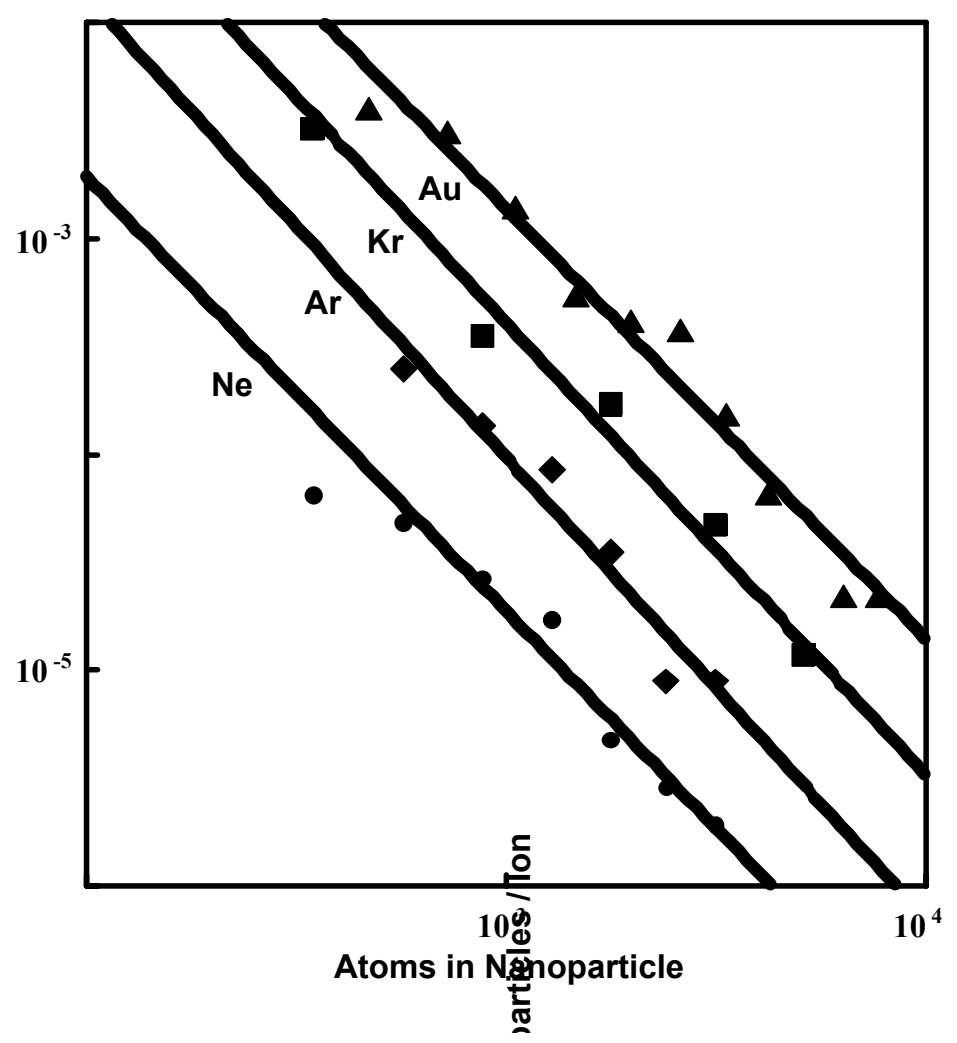

Fig. 2: Number of clusters as a function of cluster size (n) for the 400-keV Kr irradiation shown in Fig. 1, and for $400-\mathrm{keV} \mathrm{Ne}$ and Au irradiations to fluences of $6.2 \times 10^{18}$ ions $/ \mathrm{m}^{2}$ and $3.8 \times 10^{17}$ ions $/ \mathrm{m}^{2}$, respectively, and for a 500$\mathrm{keV}$ Ar irradiation to a fluence of $2 \times 10^{18}$ ions $/ \mathrm{m}^{2}$.

The size distributions that were obtained are displayed in Fig. 2. Size distributions were determined for the particles collected from the $400-\mathrm{keV} \mathrm{Kr}$ irradiation, and for $400-\mathrm{keV} \mathrm{Ne}$ and $\mathrm{Au}$ irradiations to fluences of $6.2 \times 10^{18}$ ions $/ \mathrm{m}^{2}$ and $3.8 \times 10^{17} \mathrm{ions} / \mathrm{m}^{2}$, respectively, and for a $500-\mathrm{keV}$ Ar irradiation to a fluence of $2 \times 10^{18}$ ions $/ \mathrm{m}^{2}$. Two important findings emerge from 
Fig. 2. First, the displayed power-law fits to the data from the four individual irradiations all have the same slope despite large differences in the total sputtering yield (e.g., $\sim 5$ for the Ne and $>100$ for the $\mathrm{Au}$ ). Second, the magnitude of this slope is -2 within remarkably tight error limits; the average standard deviation determined for the four runs was $<0.05$.

Early after their discovery, it was recognized [2] that a mechanism involving the cooperative motion of atoms was probably responsible for the emission of intact clusters during ion bombardment. Expanding on this concept, and noting that clusters were formed with the greatest probability under energetic heavy-ion bombardment, Bitensky and Parilis [7] formulated an analytical model for cluster emission based upon the formation and emergence of shock waves that originate from energetic subsurface collision cascades. Essentially, the arrival and reflection of a shock wave at a sample surface places the latter under tension. If this tension exceeds a critical value, known as the fracture strength, the surface fractures and fragments are ejected. The Bitensky and Parilis model predictions are in excellent agreement with the results displayed in Fig. 2. Their shock-wave model predicts a power-law decrease for the cluster size distribution with an exponent of -2 . The inverse-square dependence arises because the critical parameter in the fracture process is the energy required to create new surface area, which for a three-dimensional particle increases as its characteristic dimension squared.

The experimental verification that relatively large atom clusters, ones up to approximately 10,000 atoms, are generated during high-energy ion bombardment also offers a possible explanation for why many previously reported investigations [3-5] yielded power-law size distributions with considerably higher exponents. Very simply, a higher exponent means that smaller clusters are present with disproportionately higher probabilities in the mass spectrometry studies. Such an excess of smaller clusters is of course what would occur if all the larger clusters do not remain completely intact. As alluded to in the introduction, the very fact that the inter-atomic bond energy is so small in comparison to the energies involved in the displacement events implies that some clusters can be expected to fragment after being ablated.

We also note from the data displayed in Fig. 2 that the size of the largest nanoparticle that is ablated from the target surface increases systematically, and substantially, with increasing ion mass. Although the smallest observable particle size will depend strongly on TEM imaging conditions, and therefore can vary considerably from sample to sample, the largest particles are the easiest to observe, and the easiest to characterize. With this fact in mind, we see that some particles containing in excess of 8000 atoms were generated by the Au irradiation, while the largest for the $\mathrm{Kr}$ irradiation contained approximately 5000 atoms, and it decreased even further, to only approximately 3000 atoms, for the Ar and Ne bombardments. This increase in the largest observed nanoparticle size can be readily understood in terms of the maximum energy available in the displacement-cascade-induced shock wave, which also increases with increasing ion mass.

It might appear surprising at first that an element as ductile as Au actually fractures in the manner described by the shock-wave model of Bitensky and Parilis. To understand this, it is important to note the exceedingly fast time scale on which the shock wave develops and impacts the surface. The pressure pulse generated by a highly energetic displacement cascade is fully developed within a time less than approximately one picosecond. Traveling at the speed of sound, a criterion which can actually be exceeded by a strong shock wave, the shock wave would reach the surface from a depth of approximately $100 \AA$ also in a time of approximately one picosecond. This extremely short time scale of less than a few picoseconds is not sufficient for dislocations to either develop or move. Hence even normally highly ductile materials will become brittle at such high strain rates.

This latter observation implies that the ion-ablation technique described above should be useful for synthesizing nanoparticles of a wide variety of alloy compositions and phases, i.e., 
normally brittle materials are not required. In order to examine this concept further, we prepared a layered $\mathrm{Co} / \mathrm{Pt}$ target surface. Using the e-beam deposition procedure described in the preceding Experimental Details section, a $30 \mathrm{~nm}$ thick layer of $\mathrm{Pt}$ was deposited on $\mathrm{NaCl}$, followed by a 1.5 nm layer of Co. These materials were selected because we felt that with their large differences in atomic number (27 vs. 78 ) and crystal structure (bcc vs. fcc), our in-situ TEM technique could be used to image the CoPt layered particles that should be produced with this target. Furthermore, the selection of Pt, a highly dense material, for the substrate is consistent with the idea that highly energetic displacement cascades are needed for development of the shock waves. For a more detailed description of the dependence of nanoparticle ejection on the energy deposition please see Ref. 9, where the rate of nanoparticle ejection is shown to depend only on the location and amount of energy transferred to the target atoms.

An HRTEM micrograph of two of the larger Co/Pt particles that were synthesized is displayed in Fig. 3. The cross-fringed lattice image confirms that the collected particles are indeed three-dimensional and crystalline. Unfortunately our attempts to separate out the different layers of Pt and Co using TEM techniques have not yet proven successful; we are therefore turning to Atomic Force Microscopy in order to obtain more conclusive evidence for the existence of a layered structure. However, in situ experiments did reveal a significant change in the selected area diffraction pattern following a 20 minute anneal at $200{ }^{\circ} \mathrm{C}$, consistent with the expectation that the particles had not experienced any large thermal excursion during the shockwave induced ablation process. No change in diffraction pattern was observed during a similar prior anneal at $100{ }^{\circ} \mathrm{C}$.

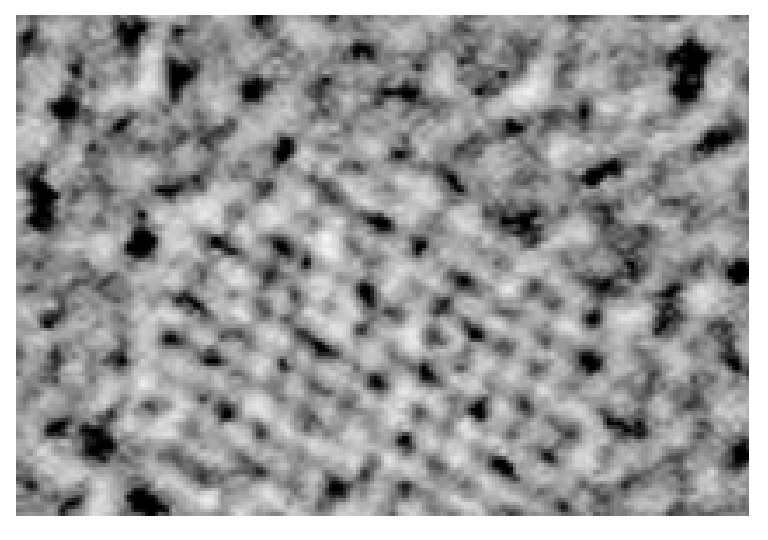

Fig. 3: High Resolution Electron Microscopy (HREM) picture of two nanoparticles from a layered $\mathrm{Cu}-\mathrm{Pt}$ target ion ablated onto a $\mathrm{C}$ grid.

Finally, we note that the ion ablation synthesis of nanoparticles discussed here produces a very broad distribution of nanoparticle sizes according to its characteristic inverse-square size distribution, from minimal clusters containing only two atoms all the way up to nanoparticles comprising thousands of atoms. Although narrow size distributions are often highly desirable for practical applications, such as in optically active devices, a broad size distribution can have advantages as well, particularly for fundamental studies of properties as a function of nanoparticle size. Our own interest centers on using in-situ TEM to determine alloy phase diagrams as a function of nanoparticle size, a task for which the ion ablation technique, and its compatibility with ultra high vacuum conditions, appears particularly well suited.

\section{SUMMARY}


TEM measurements of the larger clusters $(n \geq 500)$ that are sputtered from the surface by high-energy ion impacts reveal an inverse power-law size-distribution with an exponent of -2 that is independent of the total sputtering yield. This inverse-square dependence indicates that the larger clusters are formed when shock waves, generated by sub-surface displacement cascades, impact and ablate the surface, as predicted in the previously published model of Bitensky and Parilis [7]. An important corollary from these results is that the nanoparticles that are generated consist of simple fragments of the original surface, that is ones which have not undergone any large thermal excursion. The ion ablation technique discussed here should therefore be useful for synthesizing nanoparticles of a wide variety of alloy compositions and phases. In addition, it generates a broad distribution of nanoparticle sizes, up to approximately $10 \mathrm{~nm}$ in dimension, which should be useful for determining nanoparticle properties as a function of particle size.

\section{ACKNOWLEDGMENTS}

We wish to thank Dr. R. Csencsits for assistance with the high-resolution electron microscopy work. This research was supported by the U. S. Department of Energy, Office of Science, BES-Materials Science, under Contract No. W-31-109-Eng-38.

\section{REFERENCES}

[1] R. E. Honig, J. Appl. Phys. 29, 549 (1958).

[2] W. O. Hofer in Sputtering by Particle Bombardment III, edited by R. Behrisch and $\mathrm{K}$.

Wittmaack, (Springer, Berlin, 1991) 15.

[3] C. Staudt, R. Heinrich and A Wucher, Nucl. Instrum. and Meth. B 164165, 677 (2000).

[4] S. R. Coon, W. F. Calaway, J. W. Burnett, M. J. Pellin, D. M. Gruen, D. R. Spiegel and $\mathrm{J}$.

M. White, Surf. Sci. 259, 275 (1991).

[5] A. Wucher, M. Wahl, H. Oechsner, Nucl. Instrum. and Meth. B 82, 337 (1993).

[6] L. E. Rehn, R. C. Birtcher, S. E. Donnelly, P. M. Baldo, and L. Funk, Phys. Rev. Lett. 87. (2001).

[7] I. S. Bitensky and E. S. Parilis, Nucl. Instrum. and Meth. B 21, 26 (1987).

[8] C. W. Allen, L. L. Funk, E. A. Ryan and S. T. Ockers, Nucl. Instrum. and Meth. B 40/41, 553 (1989).

[9] R. C. Birtcher, S. E. Donnelly and S. Schlutig, Phys. Rev. Lett. 85, 4968 (2000). 
